\title{
Launching the Parametric Press
}

\author{
Matthew Conlen* \\ University of Washington
}

\author{
Fred Hohman ${ }^{\dagger}$ \\ Georgia Institute of Technology
}

\begin{abstract}
The Parametric Press is a new interactive digital magazine that aims to break down complex topics with visualization and interactive graphics. Built on open source research software, the platform acts as a bridge between theory and practice - a case study through which to improve interactive publishing tools and test visualization techniques in the wild - while empowering authors to tell data-driven stories and create explorable explanations.
\end{abstract}

Index Terms: Human-centered computing-Visualization;

\section{INTRODUCTION}

The use of visualization techniques and interactive graphics has become prevalent at major news outlets such as the New York Times and the Guardian. The information visualization research community has taken notice: news articles have been used as corpora to better understand the design space of data-driven storytelling $[6,8]$; researchers have performed controlled studies of new visualization techniques developed for public communication (e.g. [3]).

As researchers, however, we are removed from the messy constraints which drive the decision making of publishers. Similarly, research artifacts are often only evaluated in a laboratory setting, limiting our understanding of how they would be received in the wild [4]. Furthermore, while we have become accustomed to seeing high-production information graphics on the web, only a handful of well-funded newsrooms have invested in the infrastructure required to regularly produce them.

In May of 2019 we published the first issue ${ }^{1}$ of the Parametric Press: a digital magazine which utilizes data visualization and interactivity to tell stories and break down complex topics. We envisioned the magazine as a technical model for how others might implement interactive publishing platforms, a test-bed for evaluating and refining visualization tools and techniques, and an outlet for authors who want to publish this type of work but have no other place to do so.

\section{OUR MODEL}

Call for Proposals We invited writers to respond to a call for proposals for our first issue, Science + Society, focusing on examining scientific and technological phenomena that stand to shape society at large. We sought to cover topics that would benefit from using the interactive or otherwise dynamic capabilities of the web. We received 34 submissions, accepted seven, and ultimately published six articles. Authors were compensated between $\$ 500-1,000$ USD depending upon the scope of the work; the accepted authors had a variety of technical expertise, ranging from no coding experience, to professional software developers, to a particle physicist at CERN. The site infrastructure and articles were developed over the course of about three months starting in February 2019.

Collaborative Design Creating an interactive story is challenging because the medium combines prose, code, and other content such as animations. Few individuals possess the range of expertise required to develop all of this material on their own. Therefore, we did not expect authors to submit fully developed pieces. Instead, we

*e-mail: mconlen@cs.washington.edu

†e-mail: fredhohman@gatech.edu

${ }^{1}$ https://parametric.press/issue-01/ accepted idea submissions, and worked together with the authors to develop the articles, offering technical, design, and editorial assistance collectively. It is this collaborative process-the combination of individual expertise across fields and disciplines-that has allowed us to publish such a unique range of writing.

Open-source, Interactive Publishing One of the objectives of the Parametric Press is to showcase the new forms of media and publishing that are possible with tools available today, and to inspire others to create their own dynamic writings. To that end, we made available all of the software that powers the issue, including reusable components, custom data visualizations, and the publishing engine itself. It is our vision that interactive writing and data-driven storytelling will become a viable form of communication even for those outside of the largest newsrooms.

\section{Technology}

Leveraging $\mathrm{HCl}$ Research We opted to use Idyll [1], an opensource toolkit for writing interactive articles. Idyll separates editorial concerns from technical implementation details, allowing technical and non-technical contributors to efficiently collaborate. To adapt Idyll to this project's needs, we extended it to support publication of multiple posts which share common visual themes and elements, such as a branded site header and footer. Previously, Idyll only supported creating one article at a time, but the work done to support the Parametric Press has since been incorporated into the open source project. Open source research projects like Idyll afford their users the ability to create practical artifacts and perform in-the-wild evaluation [2], and software improvements made along the way can be shared for the benefit of the larger HCI community.

Anticipating Bit Rot Archiving is a major issue faced by publishers of interactive media: will the article's code still run a year from now? Ten years from now? With help from the developer of Webrecorder [5], an open source web archiving platform, we set up a system to create a digital archive of our articles at the time that they are published to the site. At the top of each Parametric Press article is an archive link that allows readers to download a WARC file, a standard format that allows it to be "played back" without requiring any web infrastructure.

\section{Critical Response}

After publishing the issue, we followed reader responses on social media and logged page views to the site. The issue has been viewed approximately 150,000 times to-date. One article, explaining the JPEG file format [7] was covered by Fast Company ${ }^{2}$. The review noted, "It's banal by now to observe that most of our mundaneseeming technology relies on mind-boggling ingenuity and complexity underneath. But its one thing to observe it and another to experience it. Thats the genius of this interactive explainer." Jeff Atwood, co-founder of Stack Overflow, wrote on Twitter, "Aw this is so cool, you can interactively corrupt a JPEG. People, this is how textbooks should have worked since I was a kid!”.

\footnotetext{
${ }^{2}$ https://www.fastcompany.com/90353987/the-secret-life-of-a-jpeg
} 


\section{ACKNOWLEDGMENTS}

Special thanks to Andrew Odewahn at O'Reilly Media, our editors Sara Stalla and Victoria Uren, and all of the authors who participated in creating the first issue. Fred Hohman is supported by a NASA Space Technology Research Fellowship.

\section{REFERENCES}

[1] M. Conlen and J. Heer. Idyll: A markup language for authoring and publishing interactive articles on the web. In ACM User Interface Software \& Technology (UIST), 2018.

[2] M. Conlen, A. Kale, and J. Heer. Capture \& analysis of active reading behaviors for interactive articles on the web. Computer Graphics Forum (Proc. EuroVis), 2019.

[3] Y.-S. Kim, K. Reinecke, and J. Hullman. Explaining the gap: Visualizing one's predictions improves recall and comprehension of data. In Proceedings of the 2017 CHI Conference on Human Factors in Computing Systems, CHI '17, pp. 1375-1386. ACM, New York, NY, USA, 2017. doi: $10.1145 / 3025453.3025592$

[4] R. Kosara and J. Mackinlay. Storytelling: The next step for visualization. Computer, 46(5):44-50, May 2013. doi: 10.1109/MC.2013.36

[5] I. Kreymer. Webrecorder-a web archiving platform and service for all (2015), 2015.

[6] E. Segel and J. Heer. Narrative visualization: Telling stories with data. IEEE transactions on visualization and computer graphics, 16(6):1139$1148,2010$.

[7] O. Shehata. Unraveling the JPEG, May 2019. doi: 10.5281/zenodo. 2655041

[8] C. D. Stolper, B. Lee, N. H. Riche, and J. Stasko. Emerging and recurring data-driven storytelling techniques: Analysis of a curated collection of recent stories. Microsoft Research, Washington, USA, 2016. 